\title{
Artificialidad y naturalidad en la discusión bioética
}

\section{The artificial and the natural in the bioethical discussion}

\author{
Lourdes Velázquez González*
}

https://doi.org/10.36105/mye.2020v31n2.03

\section{Resumen}

El problema de la manera correcta de utilizar lo artificial se ha presentado varias veces en los debates de bioética, y a menudo ha tenido que enfrentarse con un principio que siempre ha sido considerado fundamental en la ética y adoptado como criterio para evaluar la rectitud moral de las acciones humanas; es decir, el hecho de ser conformes con la naturaleza. Según este planteamiento, lo artificial es potencialmente malo, mientras que lo natural siempre es bueno. La tensión entre estos dos polos se encuentra, por ejemplo, en los debates acerca de la procreación médicamente asistida, pero emerge también en las discusiones acerca de la ética del medioambiente, de la roboética, o en los debates sobre el transhumanismo. El propósito de este artículo no se limita a tratar de mitigar el conflicto entre lo natural y lo artificial, sino que pretende afirmar que lo artificial es una parte legítima de lo natural, en cuanto que es expresión específica de la na-

\footnotetext{
* Profesora investigadora en el Centro Interdisciplinario para la Bioética de la Universidad Panamericana, Campus Ciudad de México. Vicepresidenta de la Federación Internacional de Sociedades de Filosofía (FISP). Correo electrónico: luluvela@prodigy.net.mx https://orcid.org/0000-0003-3082-8194.

Recepción: 22 de noviembre de 2019. Aceptación: 12 de enero de 2020.
} 
turaleza humana. Como ejemplo de la aplicación de esta tesis se discute el tema de la procreación médicamente asistida, demostrando que la eliminación de la objeción de artificialidad no resuelve otros problemas bioéticos en este campo, pero sí permite analizarlos con mayor claridad. Aspectos como el de los «embriones supernumerarios» o los relacionados con la legitimidad de usarlos en un contexto "heterólogo», ciertamente no son insignificantes, y muy a menudo han llegado a llamar la atención de los académicos; sin embargo, se refieren más bien a las «condiciones» o a las "consecuencias» del uso de las técnicas de reproducción médicamente asistidas, y tales dilemas éticos parecen ocupar una posición subordinada respecto del problema «preliminar» que proponemos analizar. Está claro que, si lo artificial dañara intrínsecamente la legitimidad moral de las prácticas antes mencionadas, no sería suficiente redimirlas considerando sus condiciones o consecuencias.

Palabras clave: tecnología y ética, bioética de la reproducción, sexualidad y reproducción, reproducción médicamente asistida, naturaleza humana.

\section{Introducción}

En el debate bioético periódicamente se retoma la dicotomía natu$\mathrm{ral} /$ artificial que, a menudo, asume las características de una oposición desde el punto de vista ético; es decir, que lo natural debe considerarse intrínsecamente «bueno» y lo artificial parece tener una esencia «mala»y, a lo sumo, redimible en la medida en que pueda verse como una «ayuda» a la naturaleza. Esta contraposición se ha ido extendiendo incluso dentro de la mentalidad actual, especialmente ante los temores de que la proliferación indiscriminada de productos artificiales en todos los sectores de la sociedad ha influido en las personas. Es muy fácil descubrir esa actitud anticientífica y anti-tecnológica que hoy en día está ampliamente difundida, y se basa principalmente en un miedo hacia el desarrollo 
de la tecno-ciencia que ahora parece casi fuera de control y que, siguiendo una lógica interna, arroja una sombra amenazante sobre el futuro de las generaciones venideras e incluso sobre la supervivencia de la humanidad misma. El intento de contrastar esta mentalidad ha llevado a algunos autores a tratar la ética de los agentes no humanos con un exceso tal vez de extensión de la noción de moralidad en sí (1).

Ciertamente no pretendemos abordar esta amplia gama de temas en su totalidad. Por lo que analizaremos el problema desde su raíz; es decir, omitiendo la catalogación moral que enmarca lo natural en la esfera de lo éticamente positivo y lo artificial en la de lo negativo.

Se puede resumir esta posición afirmando que en los dos ámbitos hay cosas buenas y cosas menos buenas o, si se prefiere, aspectos positivos y negativos que deben buscarse más allá de las razones del juicio moral sobre las acciones en las cuales estas dos esferas entran en contacto. Sin embargo, el propósito de este artículo es más radical; es decir, tiene la intención de mostrar que lo artificial en sí mismo es parte de la naturalidad cuando se considera en su amplio sentido; en otras palabras, cuando la «naturaleza» que se tiene en cuenta es específicamente la naturaleza humana [2]. $\mathrm{Y}$ bien, es precisamente una característica intrínsecamente sustancial de la naturaleza humana la de ser capaz o tener la intención no sólo para «someter» a la naturaleza, sino en cierta medida para «reemplazar» algunos aspectos y, de modo especial, crear junto con el mundo natural, un vasto mundo de entidades artificiales que constituyen lo que generalmente se conoce como la esfera de la «cultura». En esto se desarrolla una gama cada vez mayor de nuevos objetos materiales, pero también una serie de costumbres, tradiciones, instituciones y regulaciones, que constituyen la «forma de ser» del hombre, a diferencia de otros animales que intentan adaptarse al entorno en lugar de adaptar el entorno a sus necesidades vitales, incluso ampliando este entorno a través de una gran variedad de construcciones artificiales, ya sean materiales o inmateriales. 
Para no hacer nuestro discurso demasiado abstracto, trataremos de referirnos a un área concreta del debate bioético; a saber, a la práctica de la procreación médicamente asistida. Es fácil ver, de acuerdo con esta práctica que, si aceptamos considerar lo artificial como éticamente ilícito (excepto en los pocos casos en que una manipulación artificial facilita el cumplimiento de una función natural), nos encontramos atrapados en dilemas éticos casi insuperables. De hecho, si se afirma que las prácticas de procreación asistida, debido a su connotación artificial, son éticamente ilícitas, ya no tiene ningún sentido tratar de redimirlas, o absolver ciertos métodos mostrando indulgencia; por ejemplo, si estas prácticas se realizan en una condición «homóloga» (es decir, utilizando gametos de una misma pareja legalmente establecida) y rechazando estas prácticas en los contextos «heterólogos». Sin embargo, también en el caso de una procreación homóloga surgen pronto preguntas más detalladas y, por ejemplo, hay bioeticistas que exigen que todos los embriones obtenidos por fertilización in vitro se implanten en el útero de la mujer. Este planteamiento se presenta a menudo como una aplicación del principio del «mal menor». Sin embargo, se trata de una manera incorrecta de entender este principio, el cual nunca se puede aplicar a situaciones que ya son moralmente ilícitas; la aplicación correcta concierne sólo a diferentes opciones, pero todas intrínsecamente lícitas.

Otro ejemplo que podría considerarse es el debate sobre el uso moralmente lícito de los llamados «embriones supernumerarios» que se congelan y preservan durante las prácticas de FIV (fecundación in vitro). Sobre ellos, de hecho, hay quienes sostienen que, después de un cierto tiempo, pueden usarse para obtener células madres para su uso terapéutico o de investigación científica (lo que obviamente implica su supresión o «muerte»). Otros autores, al contrario, afirman que tal práctica es moralmente ilícita y que, por lo tanto, es correcto dejar que estos embriones mueran de muerte natural. Ante este dilema, no es raro que los partidarios del prejuicio negativo contra la artificialidad de la FIV declaren que no se 
quieren pronunciar, ya que estos embriones sobrantes «no deberían estar allí». La esterilidad de esta posición ya es un índice de la insostenibilidad de una condena ética de la FIV simplemente por su artificialidad. Eliminar este obstáculo, por el contrario, dejaría abierto sin más reservas el debate sobre muchos otros aspectos éticos de la procreación médicamente asistida que merecen mayor atención y un estudio más profundo.

\section{La cuestión de lo artificial en general}

El respeto de la naturaleza ha sido un principio fundamental de la moralidad de las acciones humanas durante la tradición que, en Occidente, ha conocido una duración de dos mil años y ha llegado a asumir una fuerza considerable, debido al hecho de que se ha incorporado a la ética cristiana y, en particular, a la ética católica.

Aparentemente, este principio se debilitó después de un proceso de «secularización» por el que atravesó la cultura occidental. Sin embargo, en las últimas décadas ha recuperado una vitalidad sorprendente, volviendo a presentarse de una forma nueva respecto a la que había asumido tradicionalmente. Hoy recibe, por ejemplo, un apoyo significativo de los que sostienen posiciones ecologistas, tanto a nivel general como particular de los planteamientos éticos (en especial en la bioética, entendida en un sentido más amplio; es decir, como reflexión que incluye no sólo el sector ético de la medicina, sino también lo que hoy se llama ética ambiental, ética animalista y sus campos relacionados).

La consecuencia de esta «valorización» de lo natural (debe entenderse en el sentido preciso de que la naturaleza no es sólo una condición de facto, sino también un «valor» que respetar) radica en una desconfianza más o menos acentuada en lo «artificial», que generalmente se juzga contraria a lo «natural». Esta oposición implica casi siempre un juicio negativo hacia lo artificial, incluso con respecto a valores que no son específicamente morales. Por ejemplo, 
cuando se dice que cierta actitud es artificial se da a entender que no es franca, genuina, auténtica, sincera. Cuando se enfatiza que un alimento es artificial, se da a entender que no tiene las características de autenticidad de los productos naturales y deja incluso la duda de que incluso puede ser dañino.

Al enfocar nuestra reflexión al ámbito moral, podemos decir que tomar la naturaleza como criterio de conducta moralmente correcto se remonta a la filosofía estoica, en la que la naturaleza era concebida como la realización inmanente de un logos; es decir, de un principio (en esencia divino, aunque no trascendente) que regulaba la conformación del mundo físico, así como el curso de los fenómenos naturales y de la existencia humana misma, de acuerdo con la racionalidad y la sabiduría. El sequere naturam, por tanto, se presentaba como un precepto ético básico que incluía la aceptación desde eventos físicos, hasta los perjudiciales, no menos que la adaptación a las condiciones sociales e históricas, que también se consideraban «naturales» en un sentido muy amplio.

Cuando el cristianismo se extendió por todo el Occidente, y debió comunicar su mensaje al mundo «pagano», era inevitable que buscara «conceptualizar» este mensaje a través de los instrumentos intelectuales y las categorías más acreditadas en ese contexto cultural. Por eso, asumieron gran parte del pensamiento neoplatónico y del estoicismo. En particular, la noción bíblico-cristiana de la creación ofrecía una especie de marco privilegiado para cristianizar la visión estoica: el logos inmanente de los estoicos se interpretaba fácilmente como la consecuencia del hecho de que el mundo expresa un orden deseado por Dios que, en su sabiduría absoluta, ha dispuesto la mejor estructura para cada cosa y de la cual también proviene la autoridad social de las personas y de las instituciones (recuérdese el dicho paulino «omnis potestas a Deo» (Romanos, XIII, 1); o sea, «todo poder proviene de Dios»). De esta manera, se estableció una especie de orden jerárquico, en cuya cima estaba Dios, Creador de la Naturaleza y, dentro y subordinado a ella, el hombre. Podríamos discutir si la visión estoica asumida 
por el cristianismo naciente estaba realmente en conformidad con el espíritu bíblico (en el cual el «Dios viviente» confirió al hombre el señorío sobre la naturaleza y «se reveló» como hombre en la historia), y en conformidad con la visión evangélica de la vida humana, inspirada por una esperanza orientada dinámicamente hacia el futuro y comprometida con la transformación del mundo y la sociedad bajo el impulso del Espíritu.

De todas maneras, tal concepción se arraigó profundamente en la cultura cristiana, y más tarde en la islámica, tanto que los pensadores medievales respaldaron siempre la tesis de que Dios «se reveló» al hombre de dos formas: a través de la Revelación propiamente dicha (su manifestación divina a los autores «inspirados» por su Espíritu) y a través de la Naturaleza. De hecho, cuando empezó a imponerse un enfoque intelectualista dentro de la teología, surgió el problema de cómo garantizar la universalidad de las normas morales, o sea su validez, para aquellos que no conocían o no aceptaban la Revelación. El camino que se abrió fue el de insistir cada vez más decisivamente sobre la capacidad humana de una comprensión de la naturaleza, accesible al intelecto humano gracias al lumen naturale (la «luz natural») que Dios mismo otorga a todos los hombres. Las premisas de esta concepción, que también podemos llamar tradicionales, son las de la admisión de un orden establecido (tanto en la naturaleza como en la sociedad), su estabilidad e inmutabilidad, y una concepción de la moralidad como forma de inserirse consciente y voluntariamente en ese orden, al que el hombre debe someterse al hacer uso de su libre albedrío. Indudablemente, en el hecho de adaptar la moral al libre albedrío radica precisamente el mal moral; y como consecuencia, la obediencia aparecía como la gran virtud fundamental: obediencia al orden natural, al orden social y al orden político constituido (3).

Este marco tradicional ha cambiado radicalmente con la llegada de la era moderna, caracterizada al menos por tres fenómenos históricos fundamentales: el nacimiento de la ciencia en el sentido mo- 
derno del término; el valor creciente atribuido a la libertad individual y el proceso de secularización.

El primer fenómeno ha implicado, entre otras cosas, el pasar del hombre de la simple técnica a la tecnología. De hecho, lo podemos considerar como una nueva rama de la técnica. La tecnología es constituida por la aplicación del conocimiento científico para satisfacer las más variadas necesidades humanas. Este fenómeno ha permitido un desarrollo prodigioso de lo artificial, la creación de algo auténticamente nuevo que se coloca al lado del mundo natural y que muy a menudo lo reemplaza. Todo esto ha traído muchos problemas que no tenemos la intención de abordar ahora. Nos limitaremos simplemente a observar que el nuevo acceso a la misma naturaleza física logrado por las ciencias experimentales implicaba un cambio radical en la forma de considerarla: como algo que puede ser «manipulado» para conocerlo más profundamente y también para poder explotarlo después de haber conocido sus secretos (la naturaleza pierde así ese carácter «sagrado», intocable e indisponible, que la tradición le atribuía). Además, el progreso de la ciencia pronto ha indicado que la naturaleza física no era algo fijo e inmutable, ya que la tierra, las formas de vida biológica, y el mismo universo habían conocido un desarrollo histórico. La constitución de las ciencias humanas, por su parte, indicaba que el mismo fenómeno de desarrollo y de variación se podía también aplicar a las formas de vida social, a las culturas, y a las costumbres de los hombres. Estos resultados del conocimiento científico se han convertido ahora en parte de la percepción común del mundo natural y humano, lo que hace difícil referirse a una naturaleza dotada de un orden intrínseco (hoy nos parece más bien como un campo de interacciones complejas entre una gran variedad de fuerzas y estructuras, que son el resultado de una historia contingente) y, aún más, como fruto de un orden inmutable.

En segundo lugar, en la visión moderna encontramos el énfasis puesto en la libertad humana, concebida ya no como una simple libertad de elección (el libre albedrio), sino como una libertad de acción que 
pertenece ante todo al individuo. La modernidad ha enfatizado esta libertad enfrentando ante todo el problema de hacerla compatible con sus limitaciones sociales, en una situación en la que incluso la noción de un orden social natural había entrado en declive y el concepto mismo de autoridad se había deteriorado. Los hombres se habían dado cuenta de que, tal como habían podido intervenir activamente en el orden natural, podían hacerlo en el orden social hasta el punto de cambiarlo radicalmente (a través de revoluciones), sin reconocer ninguna autoridad consagrada a la que obedecer y respetar de forma incondicional. En este punto, el proceso de la modernidad se acelera porque el hombre, ahora consciente de su facultad de intervenir libremente en el seno de las realidades que él mismo había «construido» (como las instituciones sociales), y al acostumbrarse poco a poco a intervenir en la misma naturaleza física, comienza a darse cuenta de que el desarrollo de las ciencias humanas (desde la medicina hasta la psicología y la sociología) le ofrecían la posibilidad de intervenir en su ser hombre, de una manera mucho más profunda que en el pasado. A este punto, el problema del respeto por la naturaleza vuelve a agudizarse. Muchos se preguntan si la libertad de acción, entendida como libertad de intervención y manipulación, puede ejercerse incluso cuando se trata de la naturaleza humana (hasta el punto de que hoy, como lo hemos mencionado, vuelve el tema de los límites de la manipulación tecnológica con respecto a la naturaleza no humana).

Tercer punto: la secularización, o más bien la pérdida de la referencia religiosa como marco básico de la concepción del mundo, del hombre y de la historia. Este fenómeno le quita a la doctrina naturalista el fundamento secular que, como hemos visto, era el fruto de la asimilación por la ética cristiana de la concepción estoica. Sin embargo, se debe reconocer que el pensamiento moderno secularizado no pudo renunciar a arraigar las doctrinas filosóficas a la naturaleza, y especialmente a la naturaleza humana, particularmente cuando se trató de garantizar sus fundamentos universales. Así, por ejemplo, se desarrolló la doctrina de la «ley natural», en 
conformidad con los intentos de establecer una «moral natural». Incluso la religión fue redimida de alguna manera en su legitimidad cuando la Ilustración teorizó una «religión natural» exenta de los autoritarismos y dogmas de las religiones institucionalizadas. Un autor agnóstico como Hume escribió un Tratado sobre la naturaleza bumana. Las Críticas kantianas son en realidad intentos de derivar los fundamentos de la gnoseología y la ética de una investigación sobre la «Razón pura»; es decir, sobre la «naturaleza» de la razón, tanto teórica como práctica, y la libertad de Kant era considerada como una característica esencial de la naturaleza humana, en la que se basa la dignidad absoluta de la persona (4).

Por lo tanto, lo que hasta aquí hemos dicho no implica que el respeto por la naturaleza no tenga significado ni relevancia desde el punto de vista moral y, de hecho, sería muy extraño que una tradición ética centenaria, que había hecho de este respeto incluso el fundamento principal de la moralidad, estuviera basada en un malentendido o en un grave error. Por otro lado, es significativo que no sólo muchos filósofos modernos hayan seguido refiriéndose a la naturaleza, sino que incluso hoy, como hemos visto, esta referencia en el campo ético resurge de una manera no trivial; véase, por ejemplo, Sowle (5).

El verdadero problema, por lo tanto, no es el de contrastar lo natural y lo artificial, demonizar a este último oponiéndolo al primero, sino el de comprender y promover la compatibilidad de estas dos dimensiones, al reconocer que es posible (y de hecho se debe) favorecer el crecimiento de lo artificial respetando la naturalidad, mientras no se absolutiza o se entiende de manera estática. El primer paso para alcanzar esta perspectiva consiste en darse cuenta de que lo artificial en sí mismo es una expresión genuina de lo natural. Se ha de tener en cuenta que el hombre mismo pertenece a la naturaleza, pero que el respeto de su constitución natural implica la aceptación de la artificialidad (aunque no en un sentido incondicional y absoluto). No es difícil dar este paso: es suficiente reflexionar sobre el hecho de que, si bien las especies vivas no hu- 
manas pueden sobrevivir y prosperar, en general, adaptándose al entorno natural, el hombre, por el contrario, sobrevive y prospera adaptando el entorno natural a sí mismo, a sus necesidades biológicas, psicológicas, sociales y espirituales. En otras palabras, desde el comienzo de su historia, el hombre ha construido su propio entorno, tanto en el esfuerzo de explotar las condiciones naturales para su ventaja como en el de defenderse de ellas y así asegurar su supervivencia física. Pero también ha encontrado los medios para satisfacer otras necesidades mediante la producción de códigos de conducta, legislaciones, estructuras políticas y administrativas, rituales y templos para el ejercicio de su religiosidad, medios de transporte, armas y ejércitos, herramientas de comunicación, etcétera.

Todo esto es artificial, producto del arte, cuando se entiende esta palabra no en el sentido moderno y posromántico de creatividad estética (o de orientación humana hacia la belleza), sino en el sentido más fundamental contenido en la noción griega de téchne, que indica un actuar y un producir efectivos, fruto de una conciencia e inteligencia de los medios adecuados para lograr ciertos fines. Este sentido más original de «arte» se expresa en la noción de técnica, que también se deriva etimológicamente de la noción de téchne. Por lo tanto, el mundo artificial es en realidad el mundo producido por el hombre gracias a la técnica que, como ya hemos visto, ha prosperado sobre todo después del desarrollo de las ciencias durante la era moderna.

En conclusión, el verdadero ecosistema del hombre es esencialmente el mundo tecnológico; un mundo que el hombre ha construido en virtud de las capacidades y necesidades intrínsecas de su naturaleza específica, de modo que el rechazo de la tecnología es equivalente a un rechazo de la «naturalidad» específicamente humana. Al decir esto, no hay intención de negar algunos hechos básicos. En primer lugar, el «mundo natural» sigue formando parte del ecosistema humano (aunque de manera «mesurada» por la tecnología) y que, por lo tanto, si un cierto respeto por la naturaleza se considera moralmente significativo, esto debe implicar tanto el respeto por la 
tecnología como el respeto por la naturaleza. En segundo lugar, que el hombre no puede evitar «adaptar» su entorno; de hecho, no sólo se adapta al entorno natural modificándolo y reemplazándolo en parte por técnicas, sino que también se adapta al entorno tecnológico que él mismo está construyendo. En tercer lugar, así como no todo lo natural es en sí mismo e incondicionalmente «bueno», lo que es artificial no es en sí mismo e incondicionalmente «malo». Sobre la base de estos elementos, es posible enmarcar los problemas éticos relacionados con la forma moralmente correcta de interactuar con la naturaleza y de desarrollar y aplicar la tecnología.

\section{Naturaleza y sexualidad}

El discurso general desarrollado anteriormente nos permite superar, en el contexto de la procreación asistida y en el que ahora queremos insistir, las objeciones preliminares que a veces se presentan debido al uso generalizado del término «técnicas artificiales» (6).

Cabe señalar, como primer punto, que estas técnicas son ampliamente comparables con las que implementa la medicina y, al menos dentro de la cultura occidental, nunca han sido condenadas moralmente. En efecto, siempre se ha entendido que estaban orientadas a «ayudar a la naturaleza», en el sentido de que promueven la recuperación de la funcionalidad biológica o biopsíquica «natural» del ser humano, tanto estimulando y fortaleciendo sus recursos naturales internos como combatiendo posibles agresiones de su entorno. Cuando se plantean objeciones a las prácticas de procreación asistida en nombre del respeto a la naturaleza, no es difícil darse cuenta de que vienen consideradas contrarias a la naturaleza humana, y perjudiciales en la relación que, en el caso del hombre, une la sexualidad y la reproducción, relación que, según esta concepción, debe ser absolutamente inseparable. Por lo tanto, todas las terapias destinadas a curar y superar la esterilidad del hombre y de la mujer serían consideradas moralmente legítimas (mientras 
quedan incluidas en la práctica médica común que se esfuerza por hacer que el hombre recupere sus habilidades reproductivas naturales) de tal modo que, si tales terapias tienen éxito, la reproducción pueda tener lugar en el contexto de un ejercicio «natural» de sexualidad, como consecuencia de una relación sexual normal. Por el contrario, si tales terapias no tienen éxito, no admitiría una procreación fuera del ejercicio «natural» de la sexualidad.

Para comprender las razones de tal posición, es necesario analizar el supuesto «biológico» en el que se basa; es decir, el supuesto de la inseparabilidad absoluta de la sexualidad y la reproducción. Este es un tema muy complejo, que se refiere a la conexión entre la corporeidad y la espiritualidad en el hombre, y que siempre ha sido una fuente de problemas para la ética (véase, por ejemplo, Moreland) (7).

Dentro del «orden natural» es bastante obvio que la sexualidad aparece como la forma según la cual muchas especies animales y vegetales (es decir, todas las especies caracterizadas por la llamada «reproducción sexuada») realizan la reproducción. En el caso de los animales, esta función reproductiva se caracteriza por la presencia de un instinto sexual que puede interpretarse como un impulso conductual dirigido a la "conservación de la especie», como una forma de ampliación del instinto de autoconservación presente en cada ser vivo. En el caso del hombre, este instinto se combina con sus dimensiones psíquicas y se expresa en el «amor sexual», entendido como una forma particular de actitud afectiva que atrae a individuos de sexos opuestos. Esta interpretación sustancialmente biológica de la sexualidad no se limitó a la tradición de la inspiración cristiana; es suficiente citar a un filósofo como Schopenhauer que consideraba el amor, incluso en su forma más ideal y romántica, como una «astucia de la voluntad» (es decir, como principio metafísico inmanente en todo lo real) que busca asegurar, a través de la reproducción, un modo de permanencia, más allá de la muerte de los singulares individuos (8). 
Siendo común en todas las tradiciones occidentales (al menos hasta el siglo XIX), la convicción de que el hombre no es un ser puramente biológico, sino también un «animal social», como ya lo señaló Aristóteles, deriva de ello que el ejercicio de la sexualidad conforme a la naturaleza humana debe también ubicarse dentro de su orden social apropiado (considerado a sí mismo como un orden natural). Esta esfera natural y social era la familia, en la cual el ejercicio correcto de la sexualidad seguía el fin reproductivo del nacimiento de seres «legítimos». Otras elaboraciones de esta doctrina «naturalista» añadieron que la familia debe ser monógama e, incluso, que la unión familiar debe ser indisoluble.

Es inútil decir que estas doctrinas no son comunes en todas las culturas y que, en particular, sólo la tradición católica ha respaldado la totalidad de estas tesis. Sin embargo, lo que nos interesa observar es que los defensores de esta doctrina sostienen que corresponde a un análisis objetivo y correcto de la naturaleza de la sexualidad humana y que las doctrinas que no aceptan uno u otro de estos elementos están equivocadas. Es precisamente en esta compleja interconexión entre sexualidad, reproducción e instituciones sociales donde se arraiga la estrecha relación entre bioética y derecho referidos a la reproducción médicamente asistida (ver, por ejemplo, Palazzani, Baldini, Casonato, Morresi, Zatti) (9). La complejidad de la regulación legal estuvo en la raíz de los referendos que se referían a las prácticas de procreación asistida (10).

Se ha objetado a estas posiciones que, mientras que en la mayoría de las especies animales el instinto sexual se activa y se manifiesta sólo en ciertos periodos; es decir, cuando la unión sexual corresponde a momentos en que el apareamiento puede ser fértil, dando lugar a la reproducción, en el hombre esta limitación no existe. Esto indicaría que en él la sexualidad no está vinculada a la reproducción ni siquiera al nivel del instinto. La respuesta dada a esta objeción se basa en un análisis adicional de la naturaleza humana en los siguientes términos: el hombre posee en la parte estrictamente animal de su naturaleza una tendencia espontánea y 
muy fuerte por la búsqueda del placer y, en particular, del placer sexual. Esta tendencia fue llamada concupiscencia. Sin embargo, el hombre es por naturaleza un animal razonable, ya que en él existe una dimensión mayor que el mero instinto; esa es la razón por la que no sólo es superior cualitativamente y en su rango jerárquico, sino que también es capaz de gobernar y controlar la parte animal vinculada a su corporeidad o instintos. Por lo tanto, la vida moral se caracteriza por una lucha constante de la razón para dominar la búsqueda del placer.

Esta es la concepción antihedonista de la moral, que ya ha encontrado sus expresiones más claras en el mundo antiguo con Sócrates y Platón, y se ha incorporado al cristianismo en la forma de la relación conflictiva entre el alma y el cuerpo y en la consiguiente doctrina del contraste entre la «ley del espíritu» y la «ley de la carne» (San Pablo, por ejemplo, habla del «stimulus carnis qui me colafizat»; es decir, del estímulo de la carne que lo azota, y que Dios impuso para mantenerlo en humildad, mientras lo somete constantemente a la prueba).

La mención de esta tendencia moralmente negativa se encuentra incluso en la definición tradicional de los fines del matrimonio cristiano: el fin primario es la procreación de hijos; el segundo objetivo es el remedium concupiscientiae: una solución para calmar los impulsos hacia lo sexual a través del ejercicio de una sexualidad intramatrimonial, pero siempre «abierta a la concepción». Sólo en tiempos más recientes en la doctrina católica se incluyó entre los propósitos del matrimonio el fin de la unión interpersonal de los cónyuges y el compromiso por el cuidado y la educación de los hijos, sin colocar estos fines en un orden jerárquico. En la superficie, esta ampliación parece implicar una mayor tolerancia respecto de la posición anterior, pero en la realidad no es así. De hecho (según esta doctrina), no se admite que sea moralmente permisible perseguir sólo uno de estos fines excluyendo a los demás: todos deben ser respetados, y esta postura determina diferentes posiciones éticas en el área que nos interesa. 
La presentación esbozada aquí se refiere aparentemente a la interpretación de la sexualidad humana en general, o a la «esfera de la sexualidad». Podríamos resumirla en la doctrina según la cual la actividad sexual se ajusta a la naturaleza humana mientras la respeta «en su conjunto», sin tomar en cuenta las circunstancias particulares y responde a sus tres fines. Sin embargo, la doctrina del respeto a la naturaleza en este campo es mucho más restrictiva, ya que se refiere a cada acto sexual considerado en sí mismo; es decir, independientemente de las interpretaciones o intenciones que lo acompañan o determinan (las circunstancias). Las consecuencias de tal doctrina son muchas y bien conocidas. La masturbación, por ejemplo, es considerada como un «acto contra la naturaleza», no sólo porque el ejercicio de la sexualidad no se ajusta al acto sexual que tiene lugar en la unión de dos cuerpos, sino también porque es totalmente ajena al propósito de la procreación. Por la misma razón las relaciones homosexuales son consideradas antinaturales. Caen bajo la misma condena casi todos los métodos anticonceptivos, ya que intencionalmente excluyen la procreación. Sólo son tolerados los métodos que consisten en explotar el conocimiento de los ritmos fisiológicos «naturales» de la mujer, limitando las relaciones a aquellos periodos en los que ésta no es fértil, pero sin inducir artificialmente esta infertilidad; el acto sexual se lleva a cabo de acuerdo con modalidades naturales, y la ausencia de fertilización se debe a circunstancias naturales. La intención puntual de no procrear es entonces moralmente aceptada porque existen razones adecuadas y porque, por otro lado, el fin de la «unión» entre cónyuges justifica las relaciones sexuales, más allá de la búsqueda pura del placer.

La problemática considerada hasta ahora podría resumirse de la siguiente manera: ninguna sexualidad sin procreación; y no es moralmente permisible separar el ejercicio de la sexualidad de la procreación porque la naturaleza lo impone; a lo máximo, se permite su uso durante periodos en los que la naturaleza no ofrece condiciones de procreación. Esta es la doctrina aún defendida por la 
Iglesia católica, y uno podría pensar que refleja la antigua concepción de la sexualidad entendida como concupiscencia -la búsqueda del placer-. Es una concepción moral que rechaza la búsqueda del placer como el motor de las acciones humanas. En otras palabras, sería una elaboración doctrinal destinada a apoyar la actitud antihedonista que caracteriza la ética tradicional y, en particular, la moral católica.

Debe reconocerse que esto es en gran medida cierto, pero el alcance de la visión naturalista se aprecia más claramente cuando se considera que, con base en ello, no sólo se condena una «sexualidad sin reproducción», sino también una «reproducción sin sexualidad», asumiendo esta segunda expresión en el sentido restrictivo de una reproducción que tiene lugar fuera de las condiciones del acto sexual «natural». Es precisamente esta interpretación restrictiva de la «naturaleza» de la sexualidad la que ha llevado a muchas oposiciones morales a todas las prácticas de procreación asistida. De hecho, queda claro que en todas éstas (con la excepción de la clonación, que no puede considerarse en un sentido apropiado como una técnica de procreación asistida), la procreación se alcanza a través de la unión de los gametos masculino y femenino; es decir, es una «reproducción sexual» en el sentido biológico del término.

La característica común de todas estas prácticas es que la fertilización no tiene lugar en el contexto del acto sexual y, por esta razón, cae bajo la condena de quienes defienden esta posición «naturalista». Por lo tanto, entendemos lo que ya hemos subrayado anteriormente: la condena moral no depende del hecho de que en estas prácticas recurramos a un cierto número de «técnicas artificiales»a menudo muy complejas (ya hemos aclarado que este tipo de posición «naturalista» no condena la técnicas médicas que «ayudan a la naturaleza» sin «reemplazarla»), sino por el hecho de que se supone que violan la naturaleza misma de la reproducción humana, que no es separable del acto sexual. Por esta razón, la Iglesia católica ha condenado desde el principio las prácticas de inseminación artificial pura y simple, incluso cuando eran «homólogas» (es decir, im- 
plementadas dentro de la pareja casada regularmente según el rito católico) y que consistía simplemente en sembrar el esperma del marido en la vagina de la esposa por medio de una jeringa. ${ }^{1}$ Está claro que este tipo de objeción es aún más radical en el caso de todas aquellas otras técnicas que involucran una fertilización extracorpórea del óvulo humano. ${ }^{2}$

Esta posición a menudo ha sido acusada de biologismo, ya que en ella la condición del hombre se equipara a la de todos los mamíferos, para los cuales el acto sexual y la reproducción son inseparables. A esta objeción teórica se agrega una dificultad para recibir del «sentido moral común» de nuestros tiempos, acostumbrados a aceptar sin problemas el uso de «técnicas» apropiadas en todas las áreas de la vida diaria y, en particular, en el campo médico. Este sentimiento común no comprende por qué una determinada técnica que permite realizar la concepción es condenable sólo porque no pasa por la mediación del acto sexual. Esto explica no sólo el hecho de que se practiquen diversas formas de procreación asistida en muchos centros y hospitales que dependen de instituciones abiertamente católicas (en las cuales, además, se respetan ciertas limitaciones morales de otro tipo), sino también el hecho de que no son pocos los autores católicos que critican abiertamente esta concepción, ya que sus defensores no pudieron mostrar de manera convincente las razones (aparte de la adhesión al esquema estoico mencionado anteriormente) por las cuales una procreación realizada fuera de las condiciones normales del acto sexual sería moralmente mala. Para hacer frente a estas dificultades, los partidarios de la doctrina naturalista han desarrollado recientemente una línea de defensa menos «biológica», enfatizando el hecho de que en el acto sexual humano se realiza una condición de amor y unión que constituye el marco apropiado para recibir a un nuevo ser; dado que en el caso de la procreación asistida no se cumple tal condición, por lo tanto, uno se enfrentaría a una procreación que no está en conformidad con la dignidad de la persona humana, tanto al hablar de la dignidad de los padres como de la del feto (véase, por 
ejemplo, Ratzinger) (11). Ahora trataremos de analizar críticamente el desarrollo de dicha doctrina naturalista.

\section{Procreación y sexualidad «humana»}

Hemos criticado previamente la idea según la cual se le da un valor absoluto a lo «natural» como motivo para condenar lo «artificial», mostrando la compatibilidad de lo «artificial» con las características específicas de la naturaleza bumana. Ahora intentaremos mostrar cómo un análisis de la sexualidad y, más específicamente, de la reproducción humana no justifican posiciones naturalistas incluso en su versión menos biológica, desde el momento en que expresan valores, los cuales se debe tener debidamente en cuenta.

Examinemos primero la cuestión de la inseparabilidad de la sexualidad y la reproducción. Si admitimos que probablemente existe dicha inseparabilidad en el caso de los animales para aclarar si subsiste en el ser humano (aunque podrían surgir dudas, considerando, por ejemplo, la práctica sexual con fines de cohesión social -sin función reproductiva- en ciertas subespecies de chimpancés), no podemos evitar hacer un análisis fenomenológico; es decir, una «descripción fiel» de lo que realmente sucede en lo que puede considerarse como un aspecto típico y específico de la sexualidad humana. Nos referimos al fenómeno del enamoramiento: esa forma de atracción entre individuos de sexo opuesto. Podemos considerar dicho fenómeno como una relación bipolar que concierne a dos personas tomadas en su totalidad y que provoca un deseo de presencia mutua, un «vivir juntos», un compartir el tiempo y la vida, que también incluye el deseo de una unión física, en la cual el deseo de llegar a la reproducción no aparece automáticamente. El enamoramiento es un sentimiento y una pasión que ciertamente tienen una componente biológica, que estimulan la esfera psíquica de los sueños, las expectativas, así como la creatividad artística y la forma de considerar la existencia en su conjunto. El hombre, 
como ser «razonable», alcanza una conciencia cuando reflexiona sobre el estado de su amor; sólo más tarde (y no necesariamente siempre) sentirá el deseo de procrear, no únicamente con la intención de «reproducirse», sino como un anhelo de tener un hijo con esa persona en particular de la cual se enamoró.

Ahondan los poetas, los novelistas, los filósofos y los psicólogos al ayudarnos a descubrir cuán profunda es la sexualidad bumana, una dimensión existencial que se presenta como una realidad omnipresente, inherente a la persona, más que una disposición biopsíquica cuya finalidad lleva a la reproducción. De ello se deduce que la inseparabilidad de la sexualidad y la reproducción (en el caso humano) carece de una justificación fenomenológica y, por lo tanto, debería basarse en argumentos rigurosos que nunca ha producido. Esto no excluye que, en muchos casos, el deseo de tener un hijo se presente como un fuerte ímpetu producto de la relación de amor entre dos seres humanos y, siendo así, que la reproducción desempeña un papel de primer orden a nivel existencial, no puramente biológico. Sin embargo, este hecho no puede tomarse como una característica necesaria.

Si lo que se ha dicho es correcto, se deduce que una sexualidad que no persigue el propósito de la procreación (pero se da cuenta de otros valores incluidos en dicha sexualidad) puede estar en conformidad con la naturaleza compleja de la sexualidad humana, así como una procreación que se realiza sin pasar a través de las condiciones biológicas de la sexualidad. Por lo tanto, estas dos formas de sexualidad humana no pueden ser condenadas moralmente por quienes defienden la conformidad con la naturaleza como criterio de moralidad y si se presentan objeciones morales, deben basarse en otros principios o valores. Supera los límites de este trabajo discutir las consecuencias de esta conclusión en lo que refiere a un ejercicio de sexualidad «sin reproducción». En cambio, estamos interesados en el alcance de los problemas relacionados con las técnicas que permiten el logro de la reproducción sin pasar por la condición fisiológica de reproducción «natural»; es decir, a través 
del acto sexual. Es por esto que ahora trataremos sólo con objeciones de tipo «naturalista», dejando de lado la discusión de otros tipos de cuestiones éticas relacionadas con la procreación asistida.

Como hemos ya visto, la posición naturalista considera dos aspectos diferentes. Cuando se trata de condenar el ejercicio del acto sexual con exclusión de la reproducción, declara que este acto contiene el propósito reproductivo en sí mismo y por ningún motivo está permitido oponerse a este fin. Esto significa que la prohibición se aplica no sólo cuando la motivación del acto es la pura búsqueda del placer (en este caso, la razón de la prohibición sería la expresión de una ética antihedonista), sino también cuando la procreación es dictada por razones médicas, económicas, sociales o psicológicas. Tampoco tal posición admitiría la justificación de la moralidad de este comportamiento por el hecho de que allí se encuentran las condiciones de unión, dedicación, intimidad y libertad que caracterizan el ejercicio auténticamente humano de la sexualidad. Aún menos aceptaría que el ser humano puede decidir libremente procrear o no procrear, cuándo y cuánto procrear, o renunciar a perseguir los aspectos «no reproductivos» de la sexualidad: esta sería la visión de una ética «libertaria», lo contrario de una ética naturalista.

Ante el hecho de que los seres humanos tienden «naturalmente» a practicar la sexualidad en todo momento -es decir, también durante los periodos no fértiles de las mujeres-, los naturalistas reconocen este ejercicio moralmente lícito, ya que corresponde a los propósitos de unión y de amor implícitos en la sexualidad humana, y eso puede llevarse a cabo siempre que el resultado reproductivo del acto sexual no se evite directamente (este acto siempre debe permanecer «abierto a la procreación»). Ahora no estamos interesados en discutir si está bien fundamentada tal perspectiva, sino en señalar que requiere que todos los aspectos de la sexualidad humana estén presentes en su ejercicio moralmente correcto. ${ }^{3}$

Consideremos ahora las prácticas de procreación asistida. En ellas, por supuesto, la reproducción es el fin primario y explícito. 
Los naturalistas «recientes» (como hemos visto) objetan que tales prácticas carecen de consistencia con la dignidad del ser humano, porque se trata de una concepción fuera del marco del amor, de la intimidad, de la unión y de la donación mutua de sus padres tal como se expresan durante el acto sexual. Sin embargo, esta forma de presentar la reproducción es quizás más retórica que objetiva si consideramos los actos sexuales individuales, ya que en la mayoría de los casos no dan lugar a concepción, incluso cuando se cumplen las condiciones más profundas del amor con la firme intención de alcanzar una concepción. Es más, muy a menudo la concepción resulta de encuentros sexuales rutinarios, o de noches en estado de ebriedad, o en el mejor de los casos sin la más mínima intención de procrear un niño -cuando no es incluso con la intención de no tenerlo-. Por lo tanto, podemos admitir que lo ideal sería que cada ser naciera libremente deseado, planeado, amado de antemano y como fruto de un acto sexual lleno de amor y donación mutua entre sus padres. Esta situación de «dignidad humana» queda en el mundo ideal; la realidad muchas veces difiere. Pero la falta de unas condiciones no puede ser razón para condenar moralmente la procreación humana y, sobre todo, la falta de la condición fisiológica, especialmente si esta falta es impuesta por la «naturaleza» y la pareja la experimenta con sufrimiento. Así sucede en el caso de una procreación asistida donde incluso se podría decir lo contrario: seguido en las prácticas de procreación asistida los aspectos bumanos de la reproducción se reúnen más que en el acto sexual natural, como veremos a continuación.

En primer lugar, la procreación de un ser constituye un fin explícito, consciente y libremente elegido, perseguido constantemente por la pareja durante todo el tratamiento. De esta manera resulta ser una cadena de acciones humanas en el sentido más amplio de la palabra; es decir, actos conscientes y libres. Además, hablando de la dignidad del bebé, parece bastante retórico decir que una atmósfera de amor debe acoger el momento de la concepción biológica; ¿no es más imprescindible una actitud de aceptación y amor mien- 
tras este niño empieza su viaje en la vida (es decir, durante el embarazo y después de su nacimiento)? Dicha condición quedaría menos garantizada en el caso de la concepción natural (que puede ser aleatoria e incluso no deseada).

En segundo lugar, parece correcto observar que el aspecto de la unión y del amor que (en casos ideales) se expresa emocionalmente e «instantáneamente» en el acto sexual, se manifiestan de manera explícita, consciente y «duradera» durante las prácticas de procreación asistida. En efecto, a veces implican un tratamiento más o menos prolongado, a veces por parte del hombre pero principalmente por parte de la mujer, que debe aceptar una intensa dosis de sufrimiento físico durante los procesos de preparación, de súper estimulación hormonal, de monitoreo, de extracción de óvulos y de transferencia de embriones; eso sin mencionar el estado de tensión y de verdadera ansiedad psicológica de que depende la incertidumbre sobre el éxito favorable de dichos tratamientos (un éxito que se sabe escaso cuando se evalúan los porcentajes).

Esta situación se agudiza cuando la pareja se ve obligada a repetir el tratamiento ante fallas seguidas (recurriendo a ellas a veces docenas de ocasiones). Para sobrellevar todas estas pruebas, no sólo se necesita un amor constante hacia el niño, deseado y buscado, sino también un apoyo mutuo y constante de parte de ambos miembros de la pareja. Por lo tanto, los padres están llamados a ser más fuertemente unidos y capaces de expresar el amor mutuo a través de la comprensión y participación que requiere esta «lucha común» (especialmente cuando los primeros intentos no tienen éxito y el tratamiento debe repetirse, con el constante sobresalto de nunca alcanzar la meta). En pocas palabras: someterse a procedimientos de procreación asistida y perseverar en ellos constituye un ejercicio de auténtica virtud: de abnegación, de aceptación del sufrimiento, de perseverancia, de espíritu de sacrificio; por lo tanto, parece bastante insultante calificar todo esto de «inmoral», sólo porque la posición naturalista ha determinado que sus prácticas no explotan las condiciones fisiológicas «naturales» (con sus característi- 
cas de placer y disfrute). Ciertamente el caso no es cambiar los planes y afirmar, por ejemplo, que la procreación asistida es moralmente «superior» en comparación con la natural. Sin embargo, nos parece correcto reconocer que esta última puede representar (al respetar otras demandas éticas que no abordaremos aqui) un ejemplo muy significativo de cómo se puede llamar al hombre a ejercer sus características morales y su dignidad de ser consciente y libre, con esas «nuevas» situaciones que lo artificial y la tecnología ponen a su disposición.

$\mathrm{Si}$, como vimos al principio, lo artificial no es más que la proyección de la naturaleza bumana, debemos reconocer que los aspectos morales de ésta pueden y deben desarrollarse sin «resignarse» a los límites y obstáculos planteados por la naturaleza humana, en su dimensión material y biológica, sino luchando por superarla «artificialmente» respetando que ésta sobrepasa los límites de la naturaleza puramente animal.

\section{Conclusiones}

Retomando el discurso general, nos hemos detenido en un análisis suficientemente detallado de la procreación médicamente asistida con un doble propósito: en primer lugar, regresarle al concepto de naturaleza la extensión, la anchura de su significado filosófico, liberándolo de los límites angostos de tipo fisicalista y materialista que lo han afectado en la cultura moderna. Según su significado más vasto, la naturaleza de un ser es el conjunto de propiedades que hacen de ese ser «lo que realmente es». Aplicado al ser humano, este criterio nos obliga a reconocer que una de sus características sobresalientes es la de construirse un mundo artificial; es decir, un sistema sumamente articulado y variado de cosas concretas, de instituciones, de formas de vida, que se colocan a un lado de aquellas condiciones de su entorno que él no ha construido y que, junto con ellas, constituyen su medioambiente real. Por lo tanto, lo artifi- 
cial es parte de la naturaleza humana y, por consiguiente, es también «natural». De esta manera el rompimiento entre natural y artificial se cierra y se elimina casi totalmente la base para afirmar que lo natural es de por sí éticamente bueno y lo artificial es tendencialmente malo o abierto al mal.

Esta posición de casi igualdad nos permite reconocer que no todo lo que es natural es «bueno», así como no todo lo artificial es «malo», en un sentido que aún no es específicamente ético sino simplemente entendido como algo positivo o negativo, favorable o dañino con respecto al hombre. En este punto es fácil ver que lo artificial es creado muchas veces por el hombre para corregir o combatir los aspectos «malos» de lo natural, como ocurre en el caso de la medicina, o cuando los hombres modifican a su beneficio el medioambiente o construyen una vasta variedad de artefactos para satisfacer sus necesidades y deseos.

Las consideraciones que acabamos de proponer aclaran que lo artificial es intrínsecamente un medio y, como tal, la licitud moral de su uso depende de los fines, las condiciones y las consecuencias de la acción humana en la cual ocurre. Con esto no se quiere afirmar que el fin, las circunstancias o las consecuencias previstas «justifican» éticamente los medios; sin embargo, se subraya que la licitud moral de un determinado acto no se puede evaluar sin tomar en cuenta todos estos aspectos. Con esto tampoco queremos negar que puedan existir actos «de por sí malos», aunque parece extremadamente difícil proponer ejemplos contundentes de tales actos (por ejemplo, hasta el matar no es en sí considerado absolutamente malo, ya que se admite una diferencia moral entre matar a un pollo y matar a una persona, y también en el caso de la persona se admite la licitud moral de matarla en contextos como la defensa propia, la pena de muerte, la guerra; es decir, cuando se consideren particulares condiciones de este acto.

En particular, un puro y simple acto, en cuanto tal, no persigue fines y sólo produce efectos. Las acciones humanas, al contrario, se caracterizan por ser actos intencionalmente realizados para perseguir 
fines representados que normalmente resultan de una selección entre los efectos del acto, según el cual ciertos fines son perseguidos y otros excluidos. La discusión detallada que hemos dedicado a la procreación médicamente asistida y a la relación entre sexualidad y reproducción ha servido para aclarar en un caso concreto este discurso. Sin embargo, esto se aplica en general a todos los casos en los cuales lo artificial sirve como medio para alcanzar ciertos fines, y estos casos son muy abundantes en la vida de las sociedades avanzadas. En cada uno de estos casos se persigue intencionalmente un fin determinado (como el desplazarse de un lugar a otro con un medio de transporte o calentar un departamento por medio de termosifones) y no se toman en cuenta (justamente) una infinidad de otros fines de las acciones humanas. Sin embargo, los efectos de los actos implicados en nuestras acciones entran en una red muy amplia y compleja de otros efectos y condiciones existentes en el mundo de la vida, produciendo como resultados no intencionales situaciones que consideramos «negativas» desde diferentes puntos de vista.

No se trata de una falta de buena voluntad o de ignorancia. Se trata del hecho de que el progreso de la ciencia y la tecnología produce sin duda la solución de muchos problemas, pero al mismo tiempo estas soluciones provocan nuevos dilemas inéditos y casi siempre imprevisibles. Por otro lado, ninguna ciencia o tecnología ofrece los instrumentos para manejar esta situación que implica juicios y opciones globales acerca del sentido, de la dirección y de las posibles limitaciones del desarrollo científico y tecnológico. En pocas palabras, es necesario disponer de un cierto abanico de fines dotados de un valor en sí mismos y no por el hecho de «servir»a algo más. Es una reflexión que nos ayudará a describir y promover estos valores la que podría ayudarnos a superar el optimismo ciego del cientificismo, así como el miedo ciego que inspira la anti-ciencia. La eco-ética, o ética del medioambiente se basa precisamente en este planteamiento, ya que no cae en la trampa y el engaño de que estos problemas se pueden solucionar únicamente mediante 
más y más tecnología. Esta toma de conciencia tiene también que acompañar nuestra forma de considerar los robots: éstos son máquinas y no dejan de serlo aunque puedan imitar al ser humano en muchas actividades y potenciar sus posibilidades. $\mathrm{Y}$ hasta ayudar a comprender mejor cómo funcionan ciertas partes de su ser (como el cerebro). Por estas razones hay que considerar con mucha prudencia las tesis del «transhumanismo» que, por lo menos según ciertos autores, afirman que se podrá alcanzar una «mejora» del hombre incorporando en su naturaleza lo artificial. Obviamente, todo depende de lo que se entiende por esta «mejora»; es decir, depende de la lista de valores que se presentará y del discurso que concretamente indique cómo las tecno-ciencias podrán ayudar el hombre a promoverlos.

\title{
Notas bibliográficas
}

\begin{abstract}
${ }^{1}$ Aunque no hay noticias documentadas, se puede considerar que las prácticas de inseminación artificial comenzaron a mediados del siglo XIX y pronto suscitaron un animado debate médico-teológico, tan es así, que a finales del siglo se planteó desde Francia a la oficina del Santo Oficio la pregunta acerca de la legalidad moral de esta práctica. La respuesta, dada en 1897, fue categórica: non licet (sin licencia). Los primeros documentos explícitos del Magisterio se remontan al periodo inmediatamente posterior a la Segunda Guerra Mundial, cuando la inseminación artificial se había generalizado: Pío XII intervino sobre el tema en 1949, 1951, 1956 y 1958, condenando la práctica absolutamente y admitiendo sólo intervenciones artificiales destinadas a facilitar el acto sexual y no a reemplazarlo por completo (se redujeron a métodos de dilatación del útero en el caso de una malformación congénita y a técnicas correctivas del acto sexual para facilitar el ascenso de la semilla en el útero femenino).

${ }^{2}$ Una presentación sistemática, autorizada y actualizada de la doctrina católica en el campo bioético está contenida en el tratado escrito por el cardenal Elio Sgreccia (12).

${ }^{3}$ Esta indivisibilidad de los aspectos unitivos y procreadores del acto sexual se subrayó inicialmente para condenar moralmente la práctica de la sexualidad sin reproducción o, concretamente, de métodos anticonceptivos y es una piedra angular teórica de la encíclica Humanae vitae (1968) de Pablo VI, en la que ni siquiera se menciona el problema de la fertilización extracorpórea in vitro, que en ese momento todavía aparecía como una especulación bastante teórica. Sólo en
\end{abstract}


1978 salió a la luz el primer bebé obtenido a través de la FIV en Cambridge, e inmediatamente después, junto con la difusión de este tipo de prácticas, las posiciones de condena comenzaron a aparecer en la literatura ética y en los documentos del Magisterio, que reanudaron y profundizaron la doctrina de la indivisibilidad de los dos aspectos del acto sexual ya enunciados por los pontífices anteriores y desarrollados en la Humanae vitae, aplicándolos a prácticas reproductivas sin sexualidad (típicas en este sentido son la encíclica Evangelium vitae de Juan Pablo II de 1995 y la Instrucción sobre el respeto por la vida humana naciente y la dignidad de la procreación, más frecuentemente citada con las palabras latinas Donum vitae, emitida por la Congregación para la Doctrina de la Fe en 1987).

En los documentos del Magisterio mencionados anteriormente, hablamos en general de dignidad sin distinguir entre la dignidad de los padres y la del niño. Así, en Donum vitae se afirma que las prácticas de fertilización artificial «no deben ser rechazadas como artificiales... sino que deben ser evaluadas moralmente en referencia a la dignidad de la persona humana, llamada a realizar la vocación divina al don del amor y el regalo de la vida « (DV Intr., 3). El niño, por lo tanto, debe ser el fruto directo del amor conyugal, no mediado por una intervención técnica, de modo que la procreación asistida, en cualquier caso, «priva a la procreación humana de la dignidad inherente a él» (DV, II, 5). Que el respeto a la dignidad del feto también puede ser violado está implícito en Evangelium vitae que, después de enfatizar que el acto generativo tiene un aspecto biológico, antropológico y teológico (n. 43), agrega que «en la biología de la generación está inscrita la genealogía de la persona». Esto implica lo que algunos autores católicos han desarrollado, o sea, que no está de acuerdo con la dignidad del niño el que en el origen de su "genealogía» no se encuentre el contexto completamente antropológico de la unión del amor entre los padres que constituyen el acto sexual.

\section{Referencias bibliográficas}

(1) FlORIDI L., SANDERS, W. The morality of artificial agents. Mind and Machines. 2004; 14(3): 349-379. https://doi.org/10.1023/B:MIND.0000035461.63578.9d

GRECO E. Genitori in provetta. Le certezze, i confini e i limiti normativi della fecondazione in vitro in Italia e nel mondo. Roma: Gremese; 2003.

(2) AGAZZI E. L'être humain et la nature dans le discours éthique. Le discours bioéthique. 2004, p. 113.

(3) RoLLIN F. Liberté responsable. Echanges. 1993; 178.

(4) KANT I. Crítica de la razón pura, trad. P. Ribas. Madrid: Taurus; 2014.

(5) SOWLE C, L.-HAKER H. (eds). Human Nature and Natural Law. Fascículo monográfico de Concilium. 2010; 3: 144. 
(6) BOMPIANI A. Le tecniche di fecondazione assistita: una rassegna critica. Milano: Edizioni Vita e Pensiero; 2006.

CAsonato C, Frosini T. La fecondazione assistita nel diritto comparato. Torino: Giappichelli; 2006. ISBN 9788834863756

(7) MoReland J.P, Scott B. Body and Soul. Human Nature and the Crisis of Ethics, Madison WI: Inter Varsity Press; 2000.

MORRESI A. Le forzature sulla procreazione assistita. Se la creatività giuridica cerca di aggirare la legge. Avvenire. 2009.

(8) SChOPEnhauer A. Metafísica del amor sexual. Buenos Aires: Goncourt; 1977.

(9) PALAZZANI L. La legge italiana sulla procreazione assistita: aspetti filosoficogiuridici. Medicina e Morale. 1999; 28(2/3): 746-760.

(10) AINIS M. I referendum sulla fecondazione assistita. Milano: Giuffrè; 2005.

BALDINI G, SOLDANO M. Tecnologie riproduttive e tutela della persona. Verso un comune diritto europeo per la bioética. Firenze: University Press; 2007. https:// doi.org/10.36253/978-88-8453-623-5

(11) RATZINGER J. El don de la vida. México: Palabra; 1992.

(12) SGRECCIA E. Manual de Bioética, Vol. I, Fundamentos y ética biomédica. Madrid: Biblioteca de Autores Cristianos; 2018. ZATTI P. Verso un diritto per la bioética. En MAZZONI, C.M (a cargo de) Una norma giuridica per la bioética. Bologna: II Mulino; 1998. 
